\title{
Design of experiments approach for ultimate strength assessment of corroded stiffened plates
}

\author{
K. Wołoszyk \\ Faculty of Mechanical Engineering and Ship Technology, Gdansk University of Technology, Gdańsk, Poland \\ Y. Garbatov \\ Centre for Marine Technology and Ocean Engineering (CENTEC), Instituto Superior Técnico, Universi- \\ dade de Lisboa, Lisboa, Portugal
}

\begin{abstract}
The impact of corrosion degradation on the ultimate strength of stiffened plates subjected to compressive loading is investigated. The DoE technique is used considering different plate and column slenderness ratios and corrosion severity. The FE method, considering geometrical and material nonlinearities, is employed. A two-stage corrosion degradation model is adopted. Firstly, a uniform thickness loss is adopted to reflect the mean loss of the volume of material. Secondly, mechanical properties are reduced, to reflect the local non-uniformity of the corroded surface, leading to a local strain reduction when compared to a smooth surface plate. As an analysis outcome, the most important governing factors and interactions between them are obtained. The post-collapse shapes are presented and discussed. The resulting structural response surface allows for fast estimation of the ultimate strength of corroded stiffened plate, revealing to be a swift and practical tool to be adopted in the engineering practice.
\end{abstract}

\section{INTRODUCTION}

Ships and offshore structures are operating in the marine environment, which is known as highly corrosive. Corrosion degradation can significantly affect structural members' strength, mainly due to reducing the initial cross-sectional area. One can identify to most common types of corrosion, pitting, and general one (Wang et al. 2014). These two types of corrosion need to be treated separately in terms of modelling and analysis. The focus of the current work is to investigate the impact of general corrosion.

General corrosion (Panayotova et al. 2010) causes metal degradation in two levels. In the first one, a uniform loss of the thickness can be considered. In the second level, the local irregularities of the corroded surface are observed. The uniform degradation model considers only the first level; thus, it is easily applied to different numerical methods and closed-form formulations. The non-uniform corrosion model is suitable only for sophisticated FE analyses. However, it was found that the non-uniformity of the corroded surface can be recorded when evaluating the mechanical properties of small-scale coupons subjected to tensile loading.

Intensive studies related to the mechanical properties of steel elements subjected to corrosion have been performed recently. (Garbatov et al. 2014) tested corroded specimens with different severity of corrosion degradation. The observed reduction of mechanical properties was significant, including yield stress, Young modulus, and total elongation. Other studies related to the mechanical properties of corroded flat specimens can be found in (Wang et al. 2017, Nie et al. 2019), and the conclusions were similar. The mechanical properties reduction was observed in severely corroded specimens and the initially 
corroded ones. However, it needs to be pointed out that if the corrosion degradation is perfectly uniform, its effect on the mean stress-strain response of the specimen will be due to the general corrosion degradation of the thickness. In this respect, (Wang et al. 2017) concluded that the reduction of mechanical properties originates from the nonuniformity of the corroded surface.

Studies related to other structural elements were also carried out. The effects of random non-linear and non-uniform time-dependent corrosion on the ultimate compressive strength of unstiffened rectangular steel plates have been analyzed by (Silva et al. 2013), where a series of 3575 corroded plate surfaces or different degree of corrosion and ages were generated, and non-linear finite element analyses were carried out. Based on a regression analysis, empirical formulae to predict strength reduction because of corrosion degradation have been derived, demonstrating good accuracy.

The ultimate strength of corroded stiffened plates was investigated experimentally by (Garbatov et al. 2017), taking into account different corrosion levels. The observed capacity reduction was significant. These experimental results have been used to validate the numerical model developed by (Woloszyk et al. 2018), where the effect of the thickness loss and subsequent mechanical properties reduction has been incorporated in the FE model. The agreement between experimental and numerical results was very good.

Series of experiments considering corroded box girders subjected to the vertical bending moment can be found in (Saad-Eldeen et al. 2011, 2014). The non-uniform corrosion loss caused significant strength reduction, but the asymmetrical structural behaviour has been observed too. The analyzed box girders aimed to model the behaviour of ship hull girders subjected to vertical bending.

In the present study, corrosion degradation on the ultimate strength of flat-bar stiffened plates is studied. Different plate and column slenderness ratios are investigated, taking into account different levels of corrosion severity. A two-stage corrosion model is adopted, including uniform thickness loss and change of mechanical properties of steel. As a function of the degree of degradation, the constitutive model of the mechanical properties is based on smallscale coupon specimens' experimental testing. As a result, the governing factors that influence the ultimate capacity of stiffened plates are identified. A polynomial formulation for assessing the residual capacity of corroded stiffened plates is established.

\section{CORROSION MODELLING}

The corrosion model used in the current study is used as presented in (Woloszyk et al. 2018), where the uniform thickness loss and mechanical properties reduction are considered coupled. The mechanical properties of mild steel change with the corrosion development in terms of the mean stress-strain response. Herein, the experimental results, as presented in (Garbatov et al. 2014), are used here. Nevertheless, only changes in yield stress and Young modulus are considered. A study presented in (Woloszyk and Garbatov 2019) showed that the total elongation and ultimate tensile stress have a marginal influence on ultimate strength and for the present study are taken equal to $400 \mathrm{MPa}$ and 0.28 , respectively. The properties are presented as a function of the Degree of Degradation (DoD), which is the percentage loss of the initial specimen volume.

However, the referred corrosion model was non-precise in the region of degradation level between $0 \%$ up to $25 \%$, which is not in line with similar studies (Wang et al. 2017, Nie et al. 2019). In the initial study presented in (Garbatov et al. 2014), the yield stress of non-corroded steel has been assumed, due to lack of intact specimens, whereas typically, this value is higher than the normative one. The model has been adjusted in (Woloszyk and Garbatov 2020a), where the initial yield stress value was based on the extrapolation from the corroded specimens. In case of Young modulus, adjustment was not needed. The relationships for Young modulus and yield stress are as follows: 


$$
\begin{gathered}
E=-1.0349 \cdot D o D+196[G P a] \\
R e=-1.7029 \cdot D o D+284.4[M P a]
\end{gathered}
$$

\section{FE MODEL}

The analyzed flat-bar stiffened plates consist of a $1.06 \mathrm{~m}$ length and $0.4 \mathrm{~m}$ wide plate and stiffeners of different heights. The structural behaviour is analyzed using the FE method employing commercial software (ANSYS 2019). SHELL181 finite elements are used, and the nonlinearities related to geometry and material are considered. The material model is defined as bilinear with hardening, as indicated in Section 2. The tangent modulus of linear hardening was considered as $E / 500$. The Newton - Raphson iterative procedure is employed to reflect the non-linear behaviour of the compressed stiffened plate. The mesh convergence studies were performed to find the optimum element size, leading to a $0.02 \mathrm{~m}$ element size (Woloszyk et al. 2018). The assumed boundary conditions are presented in Figure 1a, where the loaded edges are clamped, and unloaded edges are left free. The loading was generated as an incremental compressive load, leading to shortening of the edges, where coupled $u_{y}$ displacement was subjected.

The welding-induced initial imperfections are modelled as described in (Garbatov et al. 2016). The superposition of the global column imperfection, $c_{0}$, and local plate imperfection, $\delta_{0}$ is incorporated in the model, as presented in Figure $1 \mathrm{~b}$. The local plate distortion is a function of plate slenderness ratio $\beta$, defined as:

$$
\beta=\frac{w}{t} \sqrt{\frac{R e}{E}}
$$

where $w$ is the plate width, and $t$ is the plate thickness.

The average level of the local plate imperfections is considered as $0.1 \beta t^{2}$. In the case, the global imperfections are taken as $0.0015 l$, where $l$ is the length of the stiffened plate. The initial imperfections level is assumed to be constant for specific plate slenderness apart from the corrosion degradation level since it is strictly related to welding-induced distortion. The welding-induced distortions are applied by changing the z-coordinate on each node location of the finite element.

\section{DESIGN OF EXPERIMENT PLAN}

The ultimate strength of stiffened plates is mainly governed by two factors, i.e., the plate slenderness ratio and column slenderness ratio (Zhang and Khan 2009). The plate slenderness ratio has already been introduced in Equation 3. The column slenderness ratio is defined as:

a)

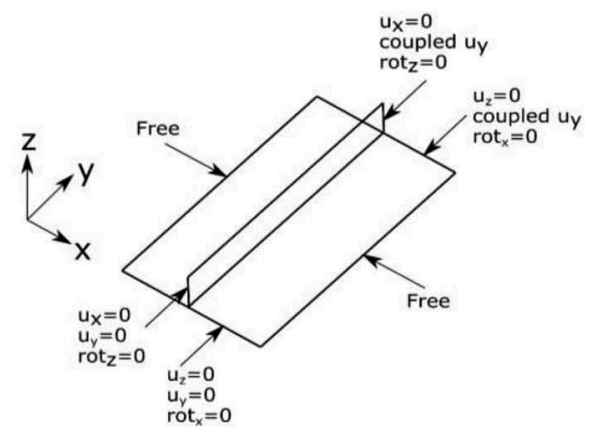

b)

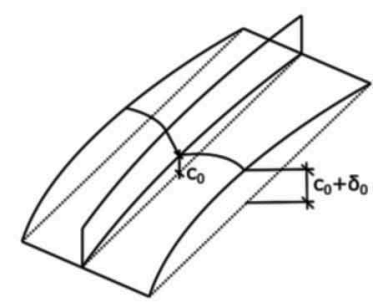

Figure 1. A) Boundary conditions and b) initial imperfections. 


$$
\lambda=\frac{l}{\pi \sqrt{I / A}} \sqrt{\frac{R e}{E}}
$$

where $I$ is the moment of inertia of the stiffened plate cross-section, $A$ is the cross-section area, and $l$ is the stiffened plate length. Based on the analysis presented in (Zhang 2015), the typical plate and column slenderness ratios are between 1.4 and 2.5 and between 0.15 and 0.8 , respectively.

Due to the non-linear relationship between ultimate strength and plate thickness, the Central Composite Design (CCD) experimental plan (Montgomery 2006) is used to investigate the dependency between the ultimate strength of corroded stiffened plates and governing factors. In that plan, the selected factors are considered taking into account their maximum $(+1)$, mean (0), and minimum (-1) values. Apart from both plate (factor A) and column (factor B) slenderness ratio, the level of corrosion degradation (factor $\mathrm{C}$ ) is considered as a third factor. The total number of 15 cases has been analyzed, considering factors values, as presented in Table 1. It needs to be noted that based on the plate and column slenderness ratios, the plate thickness $\left(t_{p}\right)$ together with stiffener dimension (height $h_{s}$ and thickness $t_{s}$ ) has been obtained. The estimated normalized ultimate strength (the ultimate force divided by the initial crosssection of the stiffener plate and yield stress value) are presented in Table 1. It could be noticed that for all studied cases, the plate thickness is equal or lower than the stiffener one. The plate dimensions and stiffener height are assumed, thus, for particular slenderness ratios, the thicknesses are resulting values.

\section{RESULTS AND DISCUSSION}

Analyzing the results presented in Table 1, one can first obtain the model's response factors' influence. More detailed information about the factorial analysis can be found in (Woloszyk and Garbatov 2020b). One of the most efficient methods is to use the half-normal probability plots (Daniel 1959). If the factor is lying on the left-hand side of the dashed line, it is nonrelevant. The three primary factors $(\mathrm{A}, \mathrm{B}, \mathrm{C})$ and interactions between them are considered $(\mathrm{A}: \mathrm{B}, \mathrm{A}: \mathrm{C}, \mathrm{B}: \mathrm{C}, \mathrm{A}: \mathrm{B}: \mathrm{C})$. Additionally, the primary factors' squares are considered too $\left(\mathrm{A}^{2}\right.$, $\left.\mathrm{B}^{2}, \mathrm{C}^{2}\right)$ to investigate the response surface's possible nonlinearity. The half-normal probability plot of the considered study is presented in Figure 2.

Table 1. Ultimate strength of stiffened plates for the DoE cases.

\begin{tabular}{rrrrrllllll}
\hline & \multicolumn{2}{l}{ Factor } & & \multicolumn{1}{l}{$t_{p}$} & $h_{s}$ & \multicolumn{1}{l}{$t_{s}$} & $\beta$ & $\lambda$ & DoD & Normalized ultimate strength \\
\cline { 2 - 9 } Case & $\mathrm{A}$ & $\mathrm{B}$ & $\mathrm{C}$ & $\mathrm{mm}$ & $\mathrm{m}$ & $\mathrm{mm}$ & - & - & $\%$ & - \\
\hline 1 & -1 & -1 & -1 & 11 & 0.25 & 13 & 1.39 & 0.15 & 0 & 0.915 \\
2 & -1 & -1 & 1 & 11 & 0.25 & 13 & 1.39 & 0.15 & 25 & 0.449 \\
3 & -1 & 1 & -1 & 11 & 0.07 & 10 & 1.39 & 0.8 & 0 & 0.846 \\
4 & -1 & 1 & 1 & 11 & 0.07 & 10 & 1.39 & 0.8 & 25 & 0.481 \\
5 & 1 & -1 & -1 & 6 & 0.25 & 8 & 2.54 & 0.15 & 0 & 0.602 \\
6 & 1 & -1 & 1 & 6 & 0.25 & 8 & 2.54 & 0.15 & 25 & 0.335 \\
7 & 1 & 1 & -1 & 6 & 0.07 & 6 & 2.54 & 0.8 & 0 & 0.64 \\
8 & 1 & 1 & 1 & 6 & 0.07 & 6 & 2.54 & 0.8 & 25 & 0.366 \\
9 & 0 & 0 & 0 & 8 & 0.1 & 9 & 1.91 & 0.47 & 12.5 & 0.566 \\
10 & -1 & 0 & 0 & 11 & 0.1 & 11.5 & 1.39 & 0.47 & 12.5 & 0.744 \\
11 & 1 & 0 & 0 & 6 & 0.1 & 7 & 2.54 & 0.47 & 12.5 & 0.507 \\
12 & 0 & -1 & 0 & 8 & 0.25 & 10 & 1.91 & 0.15 & 12.5 & 0.519 \\
13 & 0 & 1 & 0 & 8 & 0.07 & 7.5 & 1.91 & 0.8 & 12.5 & 0.55 \\
14 & 0 & 0 & -1 & 8 & 0.1 & 9 & 1.91 & 0.47 & 0 & 0.766 \\
15 & 0 & 0 & 1 & 8 & 0.1 & 9 & 1.91 & 0.47 & 25 & 0.421 \\
\hline
\end{tabular}




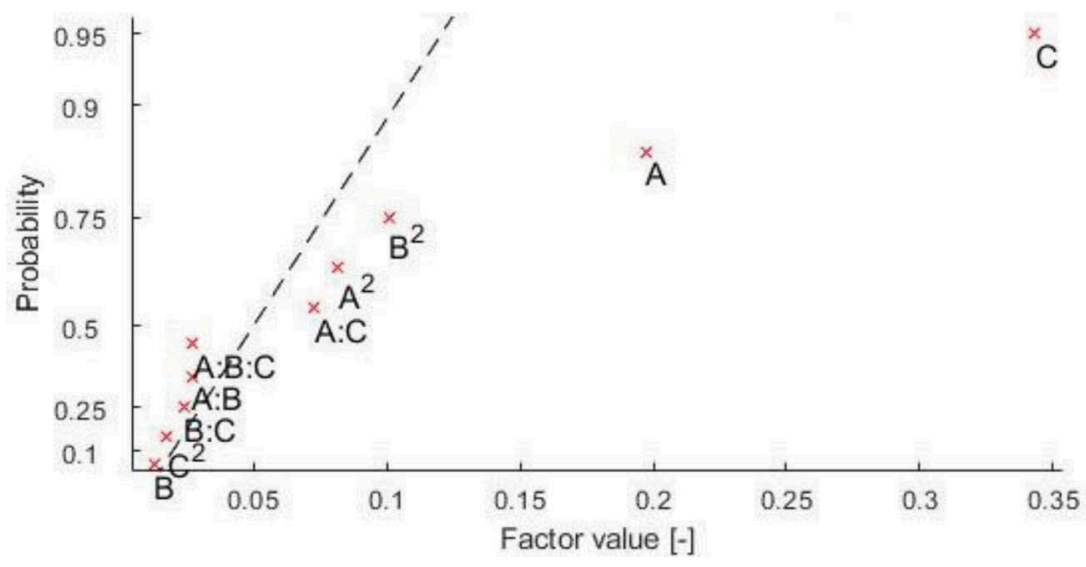

Figure 2. Half-normal probability plot.

As can be noticed, the factors that can be taken as important ones are (in the order of importance): $\mathrm{C}, \mathrm{A}, \mathrm{B}^{2}, \mathrm{~A}^{2}, \mathrm{~A}: \mathrm{C}$. The most important factor revealed to be the corrosion degradation level. Further, the plate slenderness and square of this variable significantly impact the resulting stiffened plate capacity. This indicates that the relation between that factor and the ultimate strength is non-linear. An interesting observation can be made about the column slenderness, which does not impact ultimate strength when considering only the maximum and minimum value. However, the square of this factor was revealed to be an influential one. The only interaction factor captured as necessary is between the corrosion level and plate slenderness ratio. When plate slenderness ratio is low, the decrease of the ultimate strength with corrosion development is less significant than the slenderer plates.

The different cases presented in Table 1 have different normalized ultimate strength, and the post-collapse shapes vary. In most cases, the plate induced failure lead to the collapse (e.g., case 9 - see Figure 3a). However, when the plate slenderness is significantly higher than the column one, the overall column buckling caused stiffened plate collapse (e.g., case 3 - see Figure 3b).

Based on the factors obtained from the DoE analysis, the response surface to estimate the ultimate strength of corroded stiffened plates can be established. Considering only the most essential factors, a polynomial equation may be defined as:

$$
\frac{\sigma_{U}}{R e}=0.581-0.099 \cdot x_{1}+0.041 \cdot x_{1}^{2}-0.05 \cdot x_{2}^{2}-0.172 \cdot x_{3}+0.036 \cdot x_{1} \cdot x_{3}
$$

where:

$$
x_{1}=\frac{\beta-1.965}{0.575} ; x_{2}=\frac{\lambda-0.475}{0.325} ; x_{3}=\frac{D o D-12.5}{12.5}
$$

a)

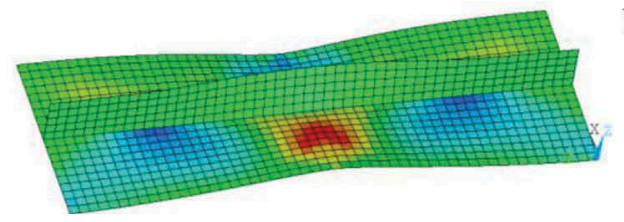

b)

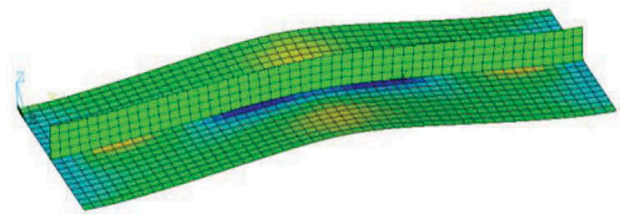

Figure 3. Post-collapse shape: a) case 9 and b) case 3 . 


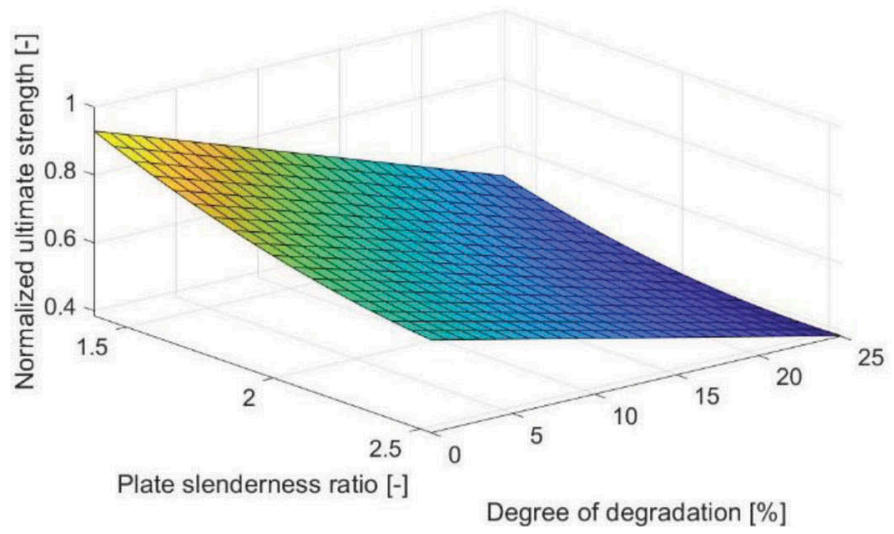

Figure 4. Response surface as a function of DoD and plate slenderness.

The response surface considering the mean value of the column slenderness ratio is presented in Figure 4. It can be noticed that the ultimate strength decreases with the increase of both plate slenderness ratio as well as corrosion degradation level.

\section{CONCLUSIONS}

The presented work investigated the influence of different governing factors on corroded stiffened plates' ultimate strength employing the DoE technique. The corrosion degradation level and plate slenderness ratio are the primary factors that govern the load-carrying capacity. The observed reduction of the ultimate strength in a severely corroded stiffened plate $(D o D=25 \%)$ reaches the level of $50 \%$ from the initial value, and it was slightly higher for slender plates. However, the column slenderness ratio was also an essential factor, considering this parameter's square. It was found that apart ultimate strength, the post-collapse shape will also vary depending on the governing factors. In the majority of the studied cases, the plate-induced failure was identified as a collapse mode. However, for the high plate slenderness ratio and low column one, the global column buckling occurred. As an analysis outcome, the response surface for fast estimation of the ultimate strength of corroded stiffened plates has been established, which may be used in the engineering practice, especially in the initial stages of the structural design process.

\section{ACKNOWLEDGEMENTS}

This work has been supported by the National Science Centre, Poland (grant No. 2018/31/N/ ST8/02380). The ANSYS software used in presented simulations in this paper was available as a part of the partnership cooperation agreement between ANSYS Inc., MESco sp. z o.o., and the Gdansk University of Technology.

\section{REFERENCES}

ANSYS, 2019. Online Manuals, Release 19.

Daniel, C., 1959. Use of Half-Normal Plots in Interpreting Factorial Two-Level Experiments. Technometrics, 1 (4), 311-341.

Garbatov, Y., Guedes Soares, C., and Masubuchi, K., 2016. Residual Stresses and Distortion in Welds. In: Reference Module in Materials Science and Materials Engineering. Elsevier.

Garbatov, Y., Guedes Soares, C., Parunov, J., and Kodvanj, J., 2014. Tensile strength assessment of corroded small scale specimens. Corrosion Science, 85, 296-303. 
Garbatov, Y., Tekgoz, M., and Guedes Soares, C., 2017. Experimental and numerical strength assessment of stiffened plates subjected to severe non-uniform corrosion degradation and compressive load. Ships and Offshore Structures, 12 (4), 461-473.

Montgomery, D.C., 2006. Design and Analysis of Experiments. John Wiley \& Sons Ltd, USA.

Nie, B., Xu, S., Yu, J., and Zhang, H., 2019. Experimental investigation of mechanical properties of corroded cold-formed steels. Journal of Constructional Steel Research, 162, 105706.

Panayotova, M. and Garbatov, Y., 2010. Corrosion of steels in marine environment, monitoring and standards. In: Safety and Reliability of Industrial Products, Systems and Structures. CRC Press, $369-413$.

Saad-Eldeen, S., Garbatov, Y., and Guedes Soares, C., 2011. Experimental assessment of the ultimate strength of a box girder subjected to severe corrosion. Marine Structures, 24 (4), 338-357.

Saad-Eldeen, S., Garbatov, Y., and Guedes Soares, C., 2014. Strength assessment of a severely corroded box girder subjected to bending moment. Journal of Constructional Steel Research.

Silva, J.E., Garbatov, Y., and Guedes Soares, C., 2013. Ultimate strength assessment of rectangular steel plates subjected to a random localised corrosion degradation. Engineering Structures, 52, 295-305.

Wang, Y., Wharton, J.A., and Shenoi, R.A., 2014. Ultimate strength analysis of aged steel-plated structures exposed to marine corrosion damage: A review. Corrosion Science, 86, 42-60.

Wang, Y., Xu, S., Wang, H., and Li, A., 2017. Predicting the residual strength and deformability of corroded steel plate based on the corrosion morphology. Construction and Building Materials, 152, $777-793$.

Woloszyk, K. and Garbatov, Y., 2019. Uncertainty assessment of ultimate strength of corroded stiffened plates subjected to maintenance. In: P. Georgiev and C. Guedes Soares, eds. Sustainable Development and Innovations in Marine Technologies. CRC Press, 429-436.

Woloszyk, K. and Garbatov, Y., 2020a. Ultimate compressive strength assessment of cleaned corroded plates with locked crack.

Woloszyk, K. and Garbatov, Y., 2020b. Analysis of Ultimate Compressive Strength of Cracked Plates with the Use of DoE Techniques. Polish Maritime Research, 27 (3), 109-120.

Woloszyk, K., Kahsin, M., and Garbatov, Y., 2018. Numerical assessment of ultimate strength of severe corroded stiffened plates. Engineering Structures, 168, 346-354.

Zhang, S., 2015. A review and study on ultimate strength of steel plates and stiffened panels in axial compression. Ships and Offshore Structures, 1-11.

Zhang, S. and Khan, I., 2009. Buckling and ultimate capability of plates and stiffened panels in axial compression. Marine Structures, 22 (4), 791-808. 